\title{
The macroscopic pancake bounce
}

Hecksher, Tina; Bro, Jonas Andersen; Jensen, Kasper Sternberg Brogaard; Larsen, Alex Nygaard; Yeomans, Julia M

Published in:

European Journal of Physics

DOI:

10.1088/1361-6404/38/1/015006

Publication date:

2017

Document Version

Publisher's PDF, also known as Version of record

Citation for published version (APA):

Hecksher, T., Bro, J. A., Jensen, K. S. B., Larsen, A. N., \& Yeomans, J. M. (2017). The macroscopic pancake bounce. European Journal of Physics, 38(1), [015006]. https://doi.org/10.1088/1361-6404/38/1/015006

\section{General rights}

Copyright and moral rights for the publications made accessible in the public portal are retained by the authors and/or other copyright owners and it is a condition of accessing publications that users recognise and abide by the legal requirements associated with these rights.

- Users may download and print one copy of any publication from the public portal for the purpose of private study or research.

- You may not further distribute the material or use it for any profit-making activity or commercial gain.

- You may freely distribute the URL identifying the publication in the public portal.

\section{Take down policy}

If you believe that this document breaches copyright please contact rucforsk@kb.dk providing details, and we will remove access to the work immediately and investigate your claim. 


\section{Home Search Collections Journals About Contact us My IOPscience}

The macroscopic pancake bounce

This content has been downloaded from IOPscience. Please scroll down to see the full text.

2017 Eur. J. Phys. 38015006

(http://iopscience.iop.org/0143-0807/38/1/015006)

View the table of contents for this issue, or go to the journal homepage for more

Download details:

IP Address: 130.226.199.186

This content was downloaded on 31/03/2017 at 12:27

Please note that terms and conditions apply.

You may also be interested in:

Transition of liquid marble impacts onto solid surfaces

C. Planchette, A. L. Biance and E. Lorenceau

Droplet impact on hydrophobic surfaces with hierarchical roughness

M A Raza, J van Swigchem, H P Jansen et al.

Macrotextured spoked surfaces reduce the residence time of a bouncing Leidenfrost drop

Colin J Patterson, Samira Shiri and James C Bird

Water spring: A model for bouncing drops

K. Okumura, F. Chevy, D. Richard et al.

Characterization of ultrahydrophobic hierarchical surfaces fabricated using a single-step fabrication methodology

S Dash, N Kumari and S V Garimella

Bouncing gel balls: Impact of soft gels onto rigid surface

Y. Tanaka, Y. Yamazaki and K. Okumura

Diagnostics for liquid dispersion due to a high-speed impact

Richard A Jepsen, Timothy O'Hern, Byron Demosthenous et al. 


\title{
The macroscopic pancake bounce
}

\author{
Jonas Andersen Bro ${ }^{1}$, Kasper Sternberg Brogaard Jensen ${ }^{1}$, \\ Alex Nygaard Larsen ${ }^{1}$, Julia M Yeomans ${ }^{2}$ and \\ Tina Hecksher ${ }^{3,4}$ \\ ${ }^{1}$ Roskilde University, PO Box 260, DK-4000 Roskilde, Denmark \\ ${ }^{2}$ Oxford University, Rudolf Peierls Centre for Theoretical Physics, 1 Keble Road, \\ Oxford OX1 3NP, UK \\ ${ }^{3}$ Centre Glass and Time, IMFUFA, Department of Science and Environment, Roskilde \\ University, PO Box 260, DK-4000 Roskilde, Denmark \\ E-mail: tihe@ruc.dk
}

Received 1 September 2016, revised 11 November 2016

Accepted for publication 21 November 2016

Published 13 December 2016

\begin{abstract}
We demonstrate that the so-called pancake bounce of millimetric water droplets on surfaces patterned with hydrophobic posts (Liu et al 2014 Nat. Phys. 10 515) can be reproduced on larger scales. In our experiment, a bed of nails plays the role of the structured surface and a water balloon models the water droplet. The macroscopic version largely reproduces the features of the microscopic experiment, including the Weber number dependence and the reduced contact time for pancake bouncing. The scalability of the experiment confirms the mechanisms of pancake bouncing, and allows us to measure the force exerted on the surface during the bounce. The experiment is simple and inexpensive and is an example where front-line research is accessible to student projects.
\end{abstract}

Keywords: water balloons, contact time, surface tension, Weber number

(Some figures may appear in colour only in the online journal)

\section{Introduction}

When a water drop hits a low friction, solid surface it typically spreads and then retracts to its original radius before bouncing. However Liu et al [2] have recently demonstrated that an impinging drop of radius $\sim 1 \mathrm{~mm}$ can leave a substrate at its maximum extension, before retracting, and therefore bounce in a pancake-like configuration (figure 1(a)). The surfaces which result in so-called pancake bouncing are arrays of hydrophobic posts of centre to centre

4 Author to whom any correspondence should be addressed. 

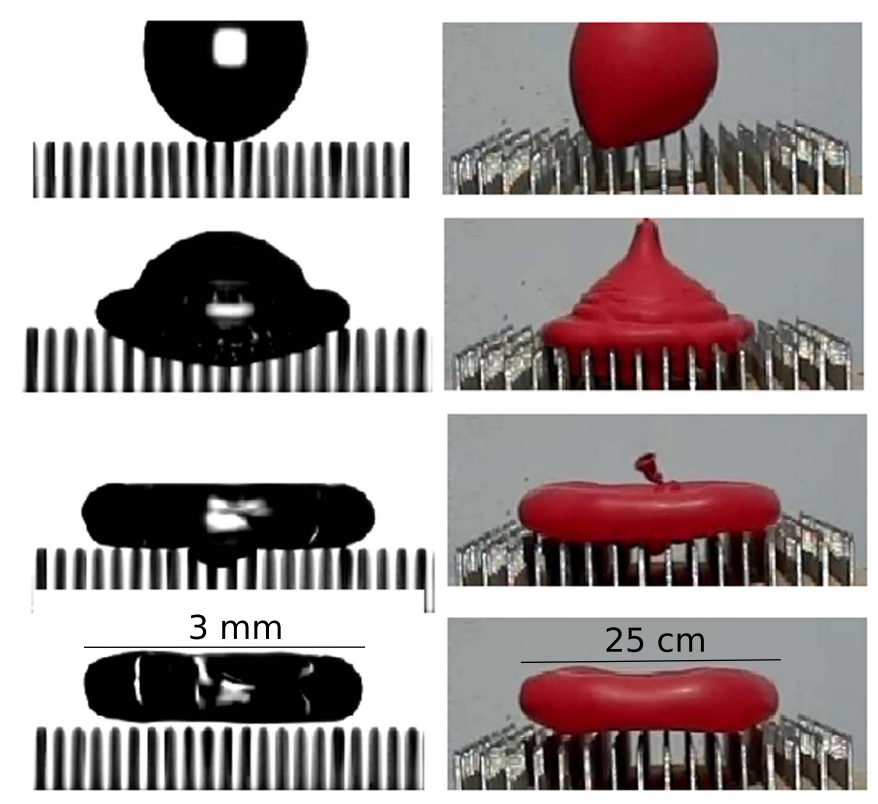

(a)

(b)

Figure 1. Rebound at maximum lateral extension (a) a millimetric droplet: the centre to centre spacing of the substrate posts is $200 \mu \mathrm{m}$, after [1]; (b) a balloon: the distance between nails is $1.85 \mathrm{~cm}$.

spacing $200 \mu \mathrm{m}$ and height $800 \mu \mathrm{m}$. Upon impact fluid is pushed between the posts, slowed, and then expelled by the hydrophobic surfaces of the posts so that the fluid entering and exiting the surface behaves like a spring. If the fluid returns to the surface while the drop is at its maximum lateral extension, and as long as it has sufficient energy, it is able to push the drop off the surface in the pancake shape.

The present paper is based on a student project carried out at Roskilde University [3] in the spring semester 2015. We asked the question: can the pancake bounce be reproduced on a larger scale? The idea was to model the water droplets with water-filled balloons, where the rubber of the balloon mimics the surface tension of the droplet, and to scale the structure of the surface accordingly. The (surprising) answer was: yes, pancake bouncing is observed for large balloons bouncing on a bed of nails at sufficiently large impact velocities (figure 1(b)). A short popular movie [4] about the experiment is available.

We show that much of the microscopic phenomenology can be recreated at macroscopic length scales. In particular we find that the contact time of the balloon with the surface is independent of the impact velocity, and we reproduce a threshold in the impact velocity below which pancake bouncing is suppressed. Moreover we are able to add to the microscopic experiments by measuring the time-dependence of the force acting on the surface as the balloon bounces.

In section 2 we describe the experimental details. Section 3 compares how water balloons bounce on a flat surface and the bed of nails, and discusses the forces exerted on the substrates by the balloons as they bounce. We then discuss the contact times in section 4, and compare the results to those obtained for millimetric water drops in [2]. Section 5 concludes the paper by summarising our results and suggests directions for further work. 


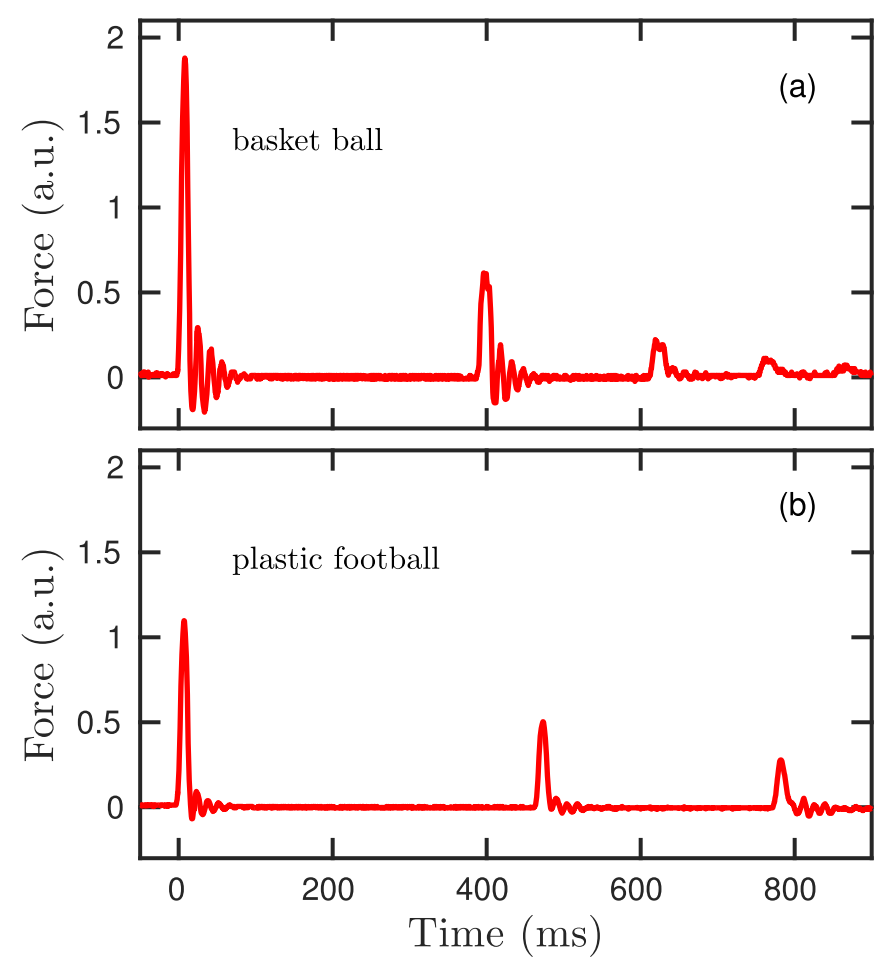

Figure 2. Example of an oscilloscope data trace from the bounce of (a) a basketball and (b) a plastic football. Impact velocity in both cases was $3.4 \mathrm{~m} \mathrm{~s}^{-1}$. The basket ball is heavier, so the impact force is relatively larger (peak is taller) than for the plastic ball. Also the subsequent ringing in the board (the oscillations seen after each impact) is more pronounced. The coefficient of restitution on the other hand is larger for the plastic ball, which is obvious from the time delay between the first and second bounce.

\section{Experimental details}

A digital reflex camera (Casio Exilim Pro EX-F1) capable of high-speed recording up to 1200 fps was used to produce movies of bouncing water balloons. For the current purpose a frame rate of $300 \mathrm{fps}$ with resolution $512 \times 384$ was sufficient to give enough details for subsequent data analysis. Some movies were also shot at $600 \mathrm{fps}$, but the image resolution is lower $(432 \times 192)$. In addition, data on the impact force were logged by an oscilloscope recording the voltage of a piezo-electric disc placed under the bounce board. Balloons were ordinary 'party balloons' purchased at the supermarket. Different types were tested and the largest available were found to perform the best. The reported experiments were carried out with balloons of radii of $6 \mathrm{~cm}$ and $4.8 \mathrm{~cm}$ when filled with water.

Two different bounce boards were used: a flat board (flat surface) and a nail board (spiked surface). The nail board was constructed to give roughly the same relation between the radius of the balloon and the distance between nails as in [2]. The nails (a total of 256) are placed in a $1.85 \mathrm{~cm} \times 1.85 \mathrm{~cm}$ square pattern.

Figure 2 shows an example of the data traces of the oscilloscope for two ordinary airfilled balls (a basket ball and a plastic football) rebounding from the flat bounce board. The two balls have the same impact velocity. The basketball is however much heavier which gives 


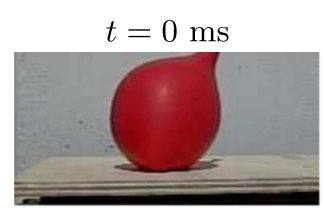

$t=133 \mathrm{~ms}$

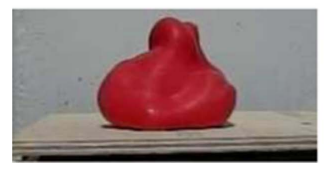

$t=33 \mathrm{~ms}$

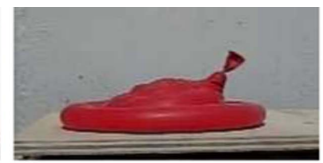

$t=167 \mathrm{~ms}$

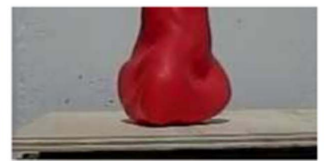

$t=67 \mathrm{~ms}$

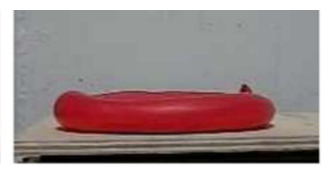

$t=200 \mathrm{~ms}$

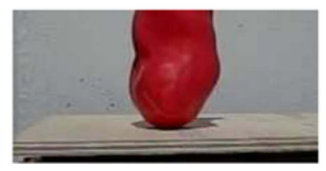

$t=100 \mathrm{~ms}$

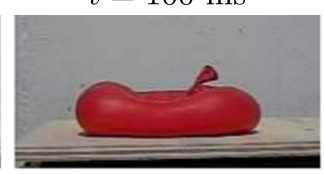

$t=233 \mathrm{~ms}$

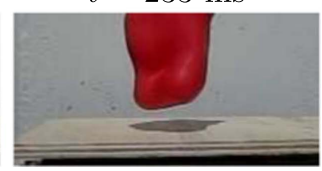

Figure 3. Waterballoon bounce on a flat surface. The snapshots show the different stages of the bounce. The first contact with the surface defines the time $t=0 \mathrm{~ms}$. The balloon detaches from the surface at $t=210 \mathrm{~ms}$. The evolution and stages of the impact and bounce are nearly identical to those observed for millimetric water droplets, except that the bounce time for the water droplets $\sim 10 \mathrm{~ms}$ (for a comparison see e.g. [8]).
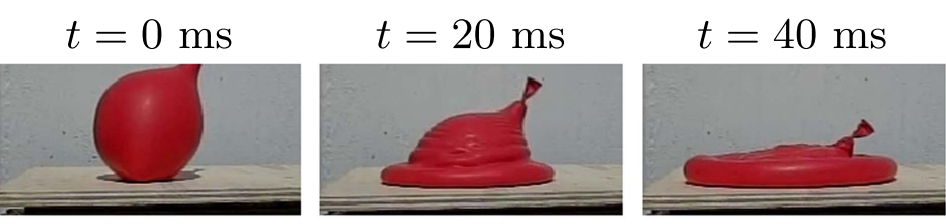

$$
t=60 \mathrm{~ms}
$$
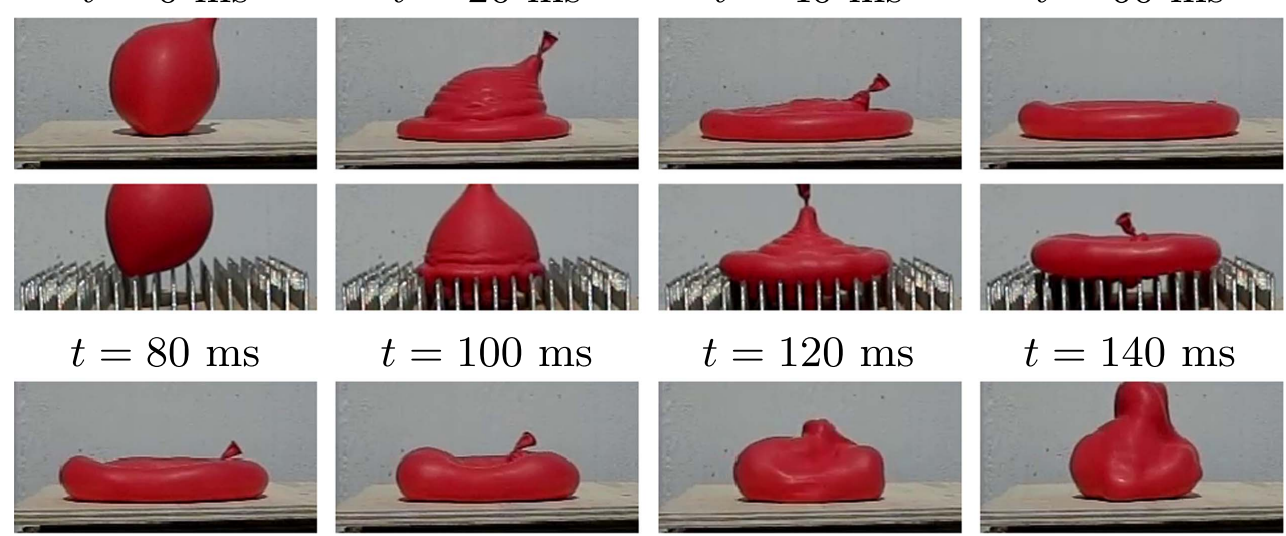

$$
t=100 \mathrm{~ms}
$$

$$
t=120 \mathrm{~ms}
$$
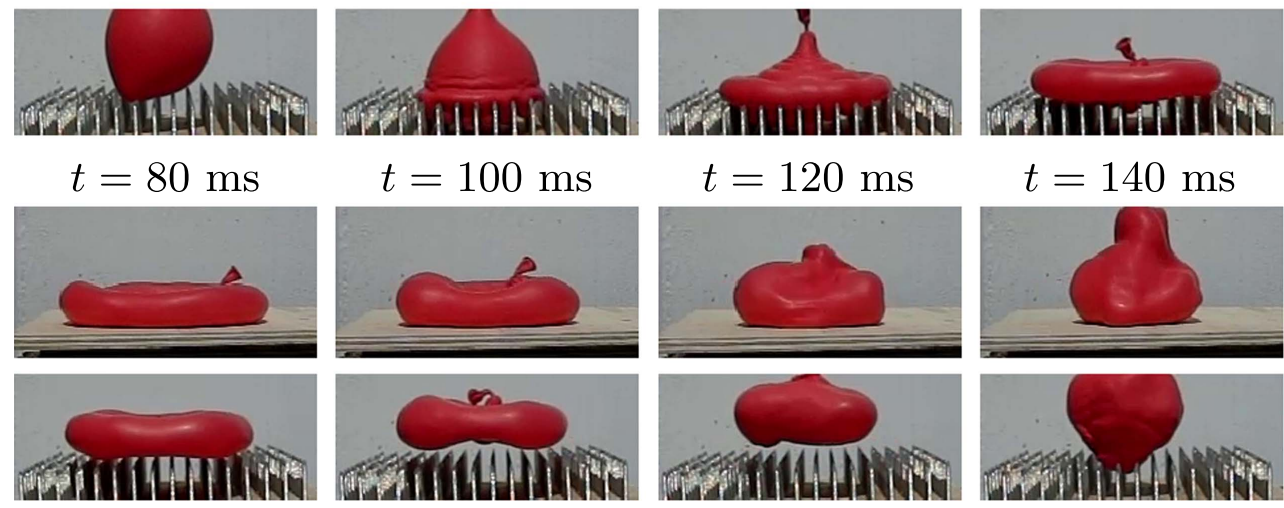

Figure 4. Comparison of a balloon bouncing on a flat surface and a spiked surface at the same impact velocity. The time evolution of the drop shape follows the same pattern in the two cases, except that the balloon detaches from the spiked surface at $65 \mathrm{~ms}$ (at the largest deformation) and then contracts in the air, while the balloon is in contact with the flat surface for much longer; it detaches at $210 \mathrm{~ms}$ after having contracted to an elongated cigar shape (compare figure 3 ).

rise to a higher peak and to more 'ringing' in the board (oscillations following the bounce). It is also immediately seen that the coefficient of restitution (COR) is smaller for the basketball since the time differences between bounces are smaller than for the plastic ball.

In order to be able to compare to droplet bouncing, we determined an effective surface tension for the balloons by inflating them and measuring the pressure, $\Delta P$. The effective surface tension $\gamma$ was then assumed to be defined by the Young-Laplace equation [5] 

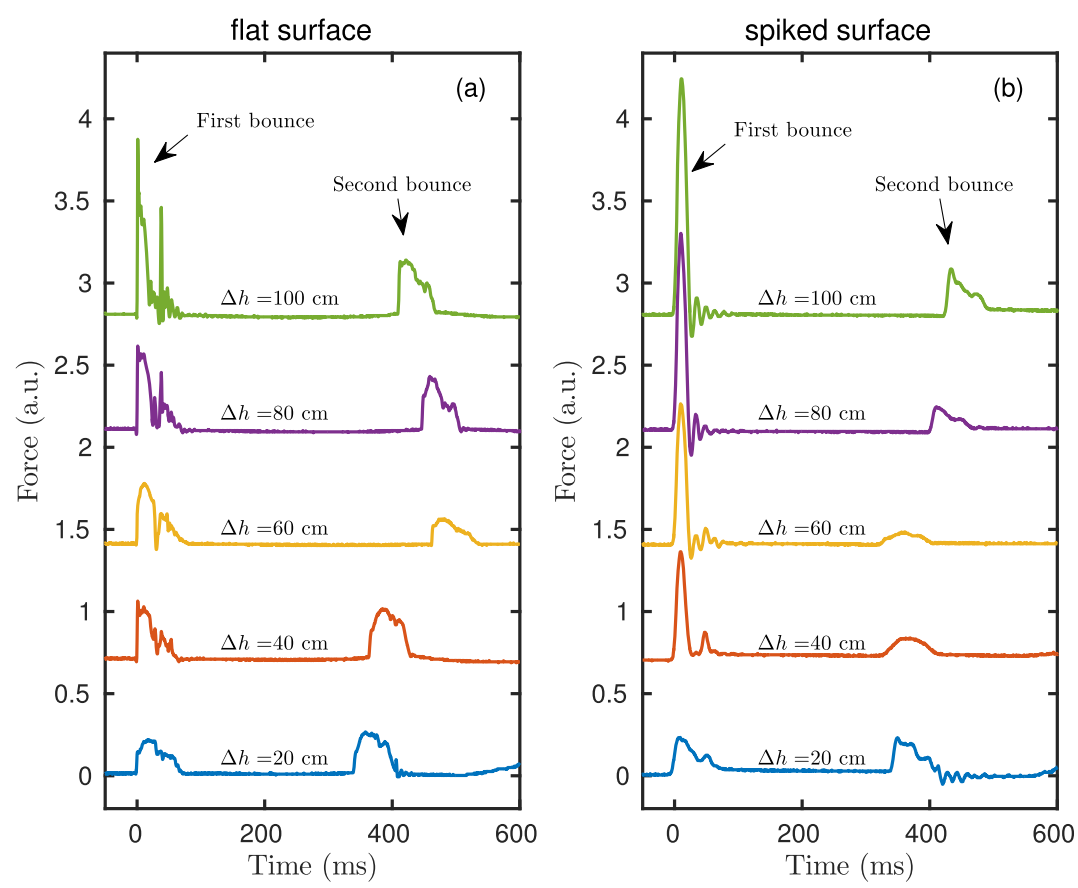

Figure 5. Series of data traces of bounces with different impact velocities (shifted on the $y$-axis for clarity). The $\Delta h$ is the height from which the balloon was dropped (same $\Delta h$ implies same impact velocity). (a) Balloon bounces on a flat surface. The impact force has an asymmetric double peak (see zoom in figure 6). (b) Balloon bounces on the spiked surface. The lowest two curves have a pronounced double-peak on first impact. From $\Delta h=60 \mathrm{~cm}$ the second peak disappears. This is the signature of pancake bouncing.

$\Delta P=\gamma \frac{2}{R}$

The pressure was measured by a U-tube manometer and the balloon radius $R$ from the circumference assuming spherical symmetry of the balloon. We obtained $\gamma=60 \pm 30 \mathrm{Nm}^{-1}$ for both the balloons at their impact radii. The large error bars are because the effective surface tension varied between balloons and depended on whether the measurement was made after the balloon was inflated or deflated to the required radius [6, 7].

\section{Results: bouncing water balloons and substrate forces}

Figure 3 shows the different stages of an impact of the $6 \mathrm{~cm}$ water balloon on a flat surface. Time $t=0$ is defined as the first contact between balloon and surface. At around $66 \mathrm{~ms}$ the balloon is maximally extended and starts retracting again, and between $200 \mathrm{~ms}$ and $233 \mathrm{~ms}$ the balloon detaches from the surface. Except for the time scale, the course of the bounce mimics closely what happens when a water droplet of size $\sim 1 \mathrm{~mm}$ impacts on a hydrophobic surface [8].

In figure 4 the bouncing of the water balloon on the flat surface and on the bed of nails are compared at the same impact velocity. The courses of the two impacts are initially similar. However, in the latter case the balloon actually makes a pancake bounce: it lifts off the bed of 


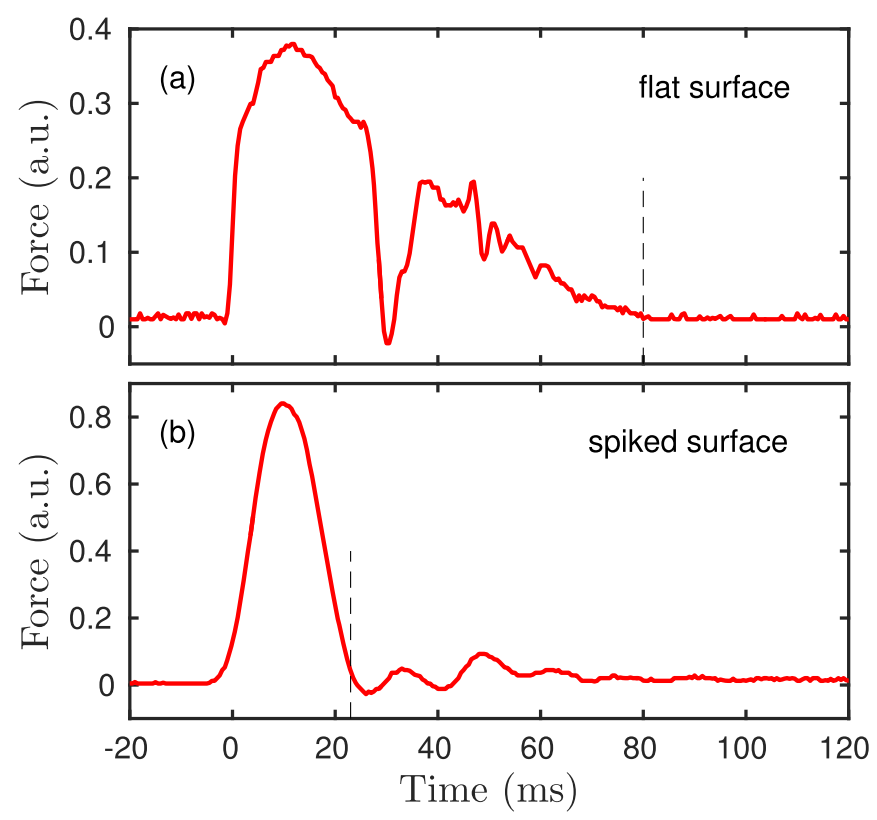

Figure 6. Zoom on first impact of (a) a normal bounce and (b) a pancake bounce with the same impact velocity (same curves as figure 5 for $\Delta h=60 \mathrm{~cm}$ ). Time when the balloon leaves the surface (equivalent to the contact time) is indicated by dashed vertical lines. Clearly, the contact time for a pancake bounce is reduced. But the shape of the peak is also markedly different: for the normal bounce there is a sharp increase in the force on impact and a double peak structure, whereas the pancake bounce corresponds to a single symmetric peak.

nails at its maximum deformation and begins to retract in the air rather than on the surface. Figure 4 also shows that the maximum extension is smaller for the impact on the spiked surface. This is because some of the material penetrates into the nail pattern instead of being pushed to the sides, and may also reflect an increased friction on the nails.

In figure 5 oscilloscope traces (equivalent to force curves) for a series of balloon bounces with different impact velocities is shown for (a) a flat surface and (b) a spiked surface. These results are for the $4.8 \mathrm{~cm}$ balloon. The normal force during the impact on a flat surface has a characteristic asymmetric double peak. There is a sharp increase as the balloon hits the surface, then the force decreases as the balloon deforms and at maximum deformation, the force is nearly zero. As the balloon starts retracting, the force increases again; the balloon pushes off the surface, and the centre of mass is accelerated in the upwards direction. Then, as the balloon leaves the balance, the force returns to zero. This behaviour is shown on an expanded scale for a balloon dropped from a height of $60 \mathrm{~cm}$ in figure $6(\mathrm{a})$.

For the spiked surface, the low velocity impacts have a similar double peak behaviour, but the shape is slightly different. This is because the impact in this case is not as abrupt: some of the balloon and mass penetrates into the nail pattern which softens the impact and gives a force curve that is less steep initially. For high impact velocities, however, there is a quantitative change: the first peak is sinusoidal in shape, and the second peak disappears (see also figure 6(b)). This behaviour of the force curves corresponds to pancake bouncing: when the material that is forced into the nail pattern recoils with sufficient energy, the balloon lifts off the surface before it retracts. 

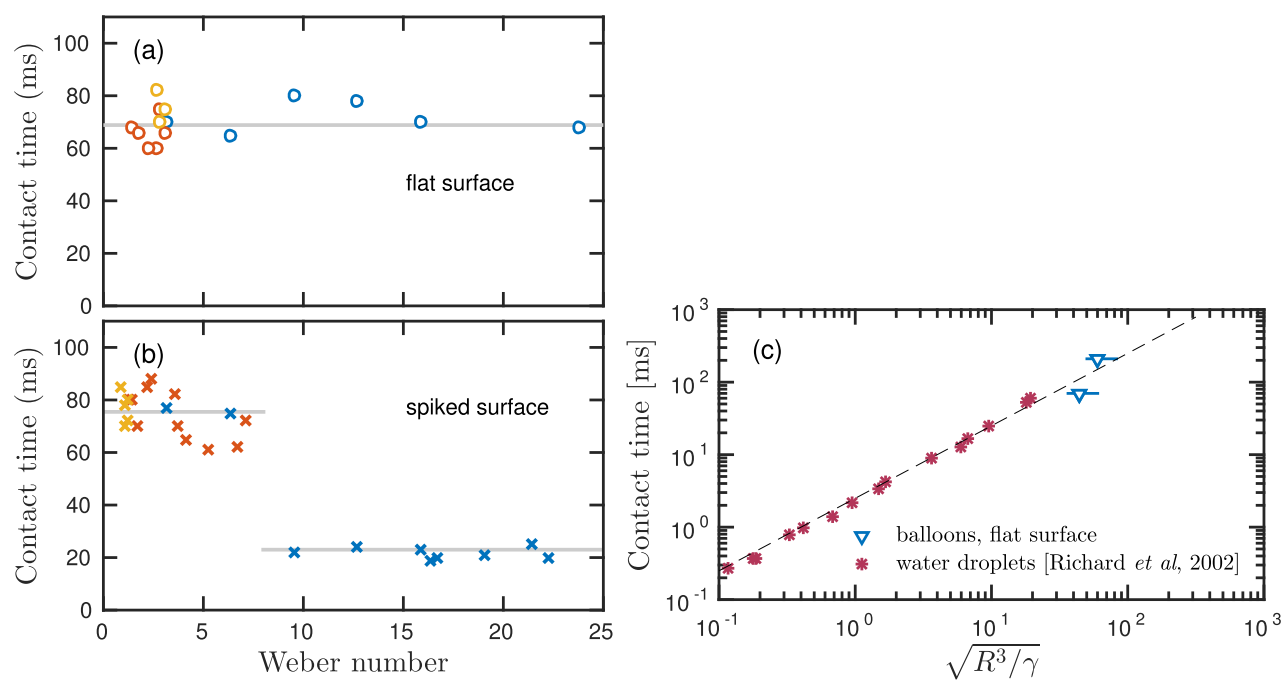

Figure 7. Contact time determined from the scope traces (see figure 6) as a function of Weber number for (a) a flat surface and (b) a spiked surface. Blue symbols are from first bounces, orange from second bounces, and yellow from third bounces (where available). For the spiked surface there is a fourfold reduction in contact time from around $80 \mathrm{~ms}$ to around $20 \mathrm{~ms}$ between $\mathrm{We}=7$ and $\mathrm{We}=10$. (c) Contact time on a flat surface compared to water droplets (data from [9]). Error bars correspond to the estimated uncertainty of the balloon surface tension.

\section{Results: contact times and comparison to water droplets}

If any contribution due to dissipation can be neglected, the expansion and contraction of the bouncing drop over the surface is controlled by a balance between inertial forces, which act to spread the drop, and surface tension, which acts to retract it. The dimensionless number which controls the ratio of inertia and surface tension is the Weber number We $=\rho v_{0}^{2} R / \gamma$, where $\rho$ is the density of water and $v_{0}$ is the impact velocity of the drop.

The contact time $t_{\text {contact }}$ is the time that the balloon (or droplet) is in contact with the surface during the bounce. On dimensional grounds ${ }^{5}$

$$
t_{\text {contact }}=c \sqrt{\rho R^{3} / \gamma}
$$

where $c$ is a numerical coefficient. Note that the contact time is expected to be independent of the impact velocity. The physics behind this is that the lateral motion during the bouncing approximates simple harmonic motion, with a period independent of the velocity amplitude. The scaling in equation (2) has been confirmed for drops on a strongly hydrophobic surface [9].

In our experiments the contact time of the bounce can be determined from visual frameby-frame inspection of the movies or from the scope traces. The contact time in the latter case is taken as the width of the impact peak: it starts at time zero when the scope registers the onset of the impact and ends when the balloons detaches and the scope again registers zero

5 The formula $t_{\text {contact }}=c \sqrt{\rho R^{3} / \gamma}$ is well established and follows from dimensional analysis. The only parameters in the problem and their dimensions are $\rho:[M][L]^{-3}, R:[L]$, and $\gamma:[M][T]^{-2}$, so $\sqrt{\rho R^{3} / \gamma}$ has dimensions of time as required. Dimensional analysis is only able to predict the form of the equation to within a constant, which we call $c$ here. 


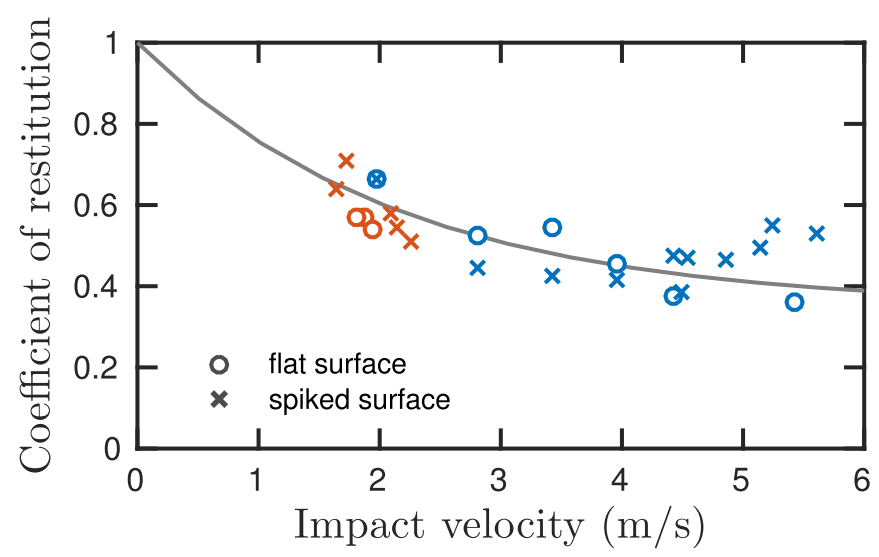

Figure 8. Coefficient of restitution defined as ratio between velocity before and after impact as a function of impact velocity. First bounces are in blue, second bounces in orange. The line is a guide to the eye.

voltage. The ringing of the board can make this a little ambiguous, however if the oscillations are centred around zero, we ascribe them to ringing; if not we assume that the balloon is still in contact with the board. The procedure is illustrated in figure 6 . The same approach can be used to obtain contact times for the second and third bounces if these are within the time window and well resolved.

In figure 7 the contact time is shown as a function of Weber number for bounces on (a) the flat surface and (b) the spiked surface for the $4.8 \mathrm{~cm}$ balloon. For the flat surface the data points all lie around an average value of approximately $70 \mathrm{~ms}$, independent of impact velocity for a fixed balloon size, (i.e. independent of Weber number) in agreement with results for water droplets [9]. For the spiked surface there is a change from a constant value of $80 \mathrm{~ms}$ at low Weber numbers to a constant value of around $20 \mathrm{~ms}$ at Weber numbers higher than $\sim 8$, marking the transition from normal bouncing to pancake bouncing. This is consistent with the behaviour observed by Liu et al [2] for a microscopic surface.

To obtain a theoretical estimate of the contact time for pancake bouncing we note that the force curve in figure 6(b) is sinusoidal. Thus the fluid penetrating the substrate is behaving as a harmonic spring. To estimate the force we assume that the balloon is pinned on the nails and stretched into a spherical cap by the downward-moving fluid. The resultant change in balloon surface area for a cap of depth $z$ is $\pi z^{2}$. To obtain the total change in area we multiply this by the number of pinning squares $\sim \pi R^{2} / d^{2}$, where $d$ is the distance between nails, giving a stored energy

$$
E=\gamma\left(\pi^{2} R^{2} / d^{2}\right) z^{2}
$$

Hence the force is

$$
F=-\gamma\left(2 \pi^{2} R^{2} / d^{2}\right) z=\rho\left(4 \pi R^{3} / 3\right) \frac{d^{2} z}{d t^{2}} .
$$

Thus a half period, the time for filling and emptying the surface, which for pancake bouncing is equivalent to the contact time, is 


$$
t_{\text {contact }}=\frac{d}{R} \sqrt{\frac{2 \pi}{3}} \sqrt{\frac{\rho R^{3}}{\gamma}}=0.56 \sqrt{\frac{\rho R^{3}}{\gamma}} .
$$

Using $R=48 \mathrm{~mm}, \gamma=60 \mathrm{~N} \mathrm{~m}^{-1}$ gives a value for the contact time of $24 \mathrm{~ms}$ in good agreement with the measured value, $20 \mathrm{~ms}$.

For this study we used only two different balloon sizes making it difficult to test if the scaling relation (2) for the contact time holds for the balloons. However, since the substance inside the balloons is water, our results should be comparable to water droplets. In [9] such data were reported as a function of drop radius. When comparing these data to the two balloon data points we have used a value of $7.2 \times 10^{-3} \mathrm{~N} \mathrm{~m}^{-1}$ for the surface tension of water. As shown in figure 7 (c), the balloon data lie nicely in continuation of the results for water droplets within the uncertainty.

Lastly, we looked at the COR of the balloon bounces. The COR is defined as the ratio between the speed immediately after and the speed immediately before the impact and is a measure of the energy loss during impact. If the impact is perfectly elastic COR is identically one, while for a perfectly inelastic impact COR is zero. As a function of impact velocity this is usually a curve that is close to one at low impact velocities then decreases to level off at a constant value at high impact velocities. In figure 8 we plot COR as a function of impact velocity which follows the expected pattern. Results from bounces on both flat surface (circles) and spiked surface (crosses) are shown and it seems that there is no significant difference between the two types of bounces.

\section{Concluding remarks}

We have studied water-filled balloons impacting on a flat surface and on a bed of nails. On flat surfaces the balloons spread, retract and then bounce with a contact time independent of the impact velocity. On the nail surface the behaviour is similar at low Weber numbers. However at high Weber numbers the balloon leaves the nails close to its maximum extension, in a pancake shape. The contact time for pancake bouncing is reduced over that for a flat surface by a factor $\sim 4$.

Force balance measurements indicate a double peaked structure for a normal bounce, with maxima associated with the the drop hitting and leaving the surface. For a pancake bounce there is a single, symmetric peak of a sinusoidal form. We argue that the harmonic force results from the balloon being pushed down between the posts by the impacting fluid, and then acting as a spring to launch the drop before retraction.

The behaviour of the balloons is surprisingly similar to that of millimetric bouncing drops, but with timescales longer by a factor $\sim 10$. In particular pancake bouncing has been observed for substrates patterned with hydrophobic posts with a similar reduction in the contact time. However, here the spring force is provided by the hydrophobic covering of the posts which tends to decelerate and then eject fluid entering the surface.

The experiment is accessible to undergraduate students in terms of expertise, cost and understanding. In the future it might be of interest to probe the analogy between water droplets and water filled balloons in more detail by considering a greater range of balloon dimensions or higher Weber numbers, when drops break up upon bouncing but balloons cannot. 


\section{References}

[1] Moevius L, Liu Y, Wang Z and Yeomans J M 2014 Langmuir 3013021

[2] Liu Y, Moevius L, Xu X, Qian T, Yeomans J M and Wang Z 2014 Nat. Phys. 10515

[3] Larsen A, Bro J and Sternberg K 2015 Student Report Roskilde University http://rudar.ruc.dk/ handle/1800/ 24942

[4] Pancake bouncing balloons, 2015 https://youtube.com/watch?v=hpsnxFhEgTI

[5] Lautrup B 2005 Physics of Continous Matter (Bristol: Institute of Physics Publishing)

[6] Osborne W A and Sutherland W 1909 Proc. R. Soc. B 81485

[7] Hermans L J F (Jo) 2010 Europhys. News 4131

[8] Clanet C, Beguin C, Richard D and Quéré D 2004 J. Fluid. Mech. 517199

[9] Richard D, Clanet C and Quéré D 2002 Nature 417811 